\title{
Filtering in Gaussian Linear Systems with Fuzzy Switches
}

\author{
Zied Bouyahia, Stéphane Derrode, and Wojciech Pieczynski
}

\begin{abstract}
This work extends recent results on Conditionally Gaussian Observed Markov Switching Models (CGOMSM) by incorporating fuzzy switches in the model, instead of hard ones. This kind of generalization is of interest for applications involving continuous switching regimes, such as tracking an object using cameras in intermittent sunlight and shadow conditions. The filter developed hereby is recursive, optimal and exact, up to an approximation of integrals according to some fuzzy measure. Experiences on simulated and on real data -dealing with outdoor air temperature and power consumption of a building- confirm the accuracy and effectiveness of the proposed filter compared to the hard filter with 'crisp' switches.
\end{abstract}

Index Terms-Triplet Markov models, Fuzzy switching linear model, Fast filtering.

\section{CMSHLM}

\section{NomenClature}

CGMSM

CGOMSM

$\boldsymbol{X}_{1}^{N}$

$X_{n}, x_{n}$

$\boldsymbol{X}_{1}^{N}, \boldsymbol{Y}_{1}^{N}, \boldsymbol{R}_{1}^{N}$

$\boldsymbol{Z}_{n}, \boldsymbol{T}_{n}$

K

$m, q$

$\mathbb{E}\left[X_{n} \mid \boldsymbol{Y}_{1}^{n}=\boldsymbol{y}_{1}^{n}\right]$

$\boldsymbol{M}_{n}\left(r_{n}\right)$

$\nu \otimes \nu$

Conditionally Markov switching hidden linear model.

Conditionally Gaussian Markov switching Model.

Conditionally Gaussian observed Markov switching model, a CGMSM where (5 holds.

Conditionally Gaussian observed fuzzy Markov switching model, a CGOMSM with fuzzy switches.

A stochastic process of size $N$.

A random variable at time index $n$, and a realization.

State, observation and switches (also called jump) processes, respectively.

Denotes $\left(X_{n}, Y_{n}\right)^{\top}$ and $\left(X_{n}, R_{n}, Y_{n}\right)^{\top}$, respectively.

Number of switches.

Dimension of the states and observations. Filter at time index $n$ (also denoted $\left.x_{n}\left(\boldsymbol{y}_{1}^{n}\right)\right)$.

Denotes $\mathbb{E}\left[\left(X_{n}, Y_{n}\right)^{\top} \mid r_{n}\right]$.

Fuzzy measure on $[0,1]$ used, where $\delta$ is the Dirac mass, and $\mu$ the Lebesgue measure.

Denotes the product of measures.

\section{INTRODUCTION}

Let us consider the problem of statistical optimal filtering in the presence of switches. Three stochastic sequences are involved: states $\boldsymbol{X}_{1}^{N}=\left(X_{1}, \ldots, X_{N}\right)$, switches $\boldsymbol{R}_{1}^{N}=\left(R_{1}, \ldots, R_{N}\right)$, and observations $\boldsymbol{Y}_{1}^{N}=\left(Y_{1}, \ldots, Y_{N}\right)$. For each $n=1, \ldots, N$, the random variables $X_{n}$ and $Y_{n}$ take their values in $\mathbb{R}^{m}$ and $\mathbb{R}^{q}$

Z. Bouyahia is with College of Arts and Applied Sciences, Dhofar University, Salalah, Oman. e-mail: zbouyahia@du.edu.om. S. Derrode is with Université de Lyon, CNRS, École Centrale de Lyon, LIRIS, CNRS UMR 5205, France. e-mail: stephane.derrode@ec-lyon.fr. Wojciech Pieczynski is with Telecom SudParis, SAMOVAR, CNRS UMR 5157, Institut Polytechnique de Paris, France. e-mail: wojciech.pieczynski@telecom-sudparis.eu

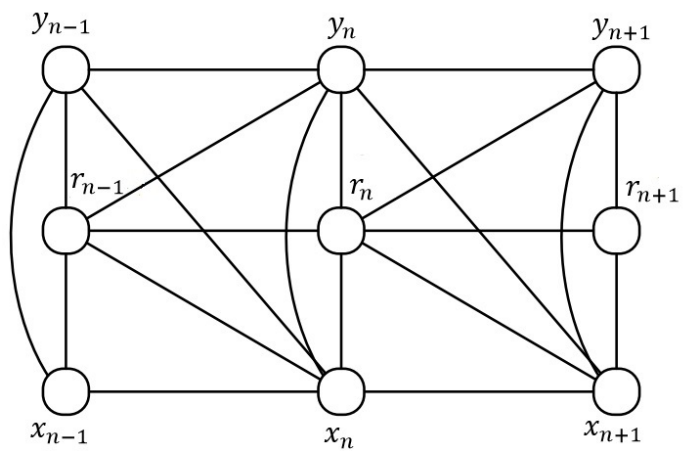

Fig. 1. Dependence graph of conditionally Gaussian observed Markov switching model.

respectively, while $R_{n}$ takes its values in the finite discrete set $\Omega=\{0,1, \ldots, K-1\}$. For the sake of simplification, we will assume in the remainder of this paper that (i) $m=q=1$, i.e. $\boldsymbol{X}_{1}^{N}$ and $\boldsymbol{Y}_{1}^{N}$ are scalar-valued processes, and (ii) that $K=2$. We consider these hypotheses only to simplify the presentation of the filtering method; the algorithms proposed in the following can be extended to the cases of vectorial processes and for a number of switches greater than two.

The problem is to sequentially estimate each $X_{n+1}$ from $\boldsymbol{Y}_{1}^{n+1}$ Fast recursive optimal filters compute the estimated $\hat{x}_{n+1}\left(\boldsymbol{y}_{1}^{n+1}\right)=$ $\mathbb{E}\left[X_{n+1} \mid \boldsymbol{Y}_{1}^{n+1}=\boldsymbol{y}_{1}^{n+1}\right]$ from $\hat{x}_{n}\left(\boldsymbol{y}_{1}^{n}\right)$ and $y_{n+1}$. The 'Conditionally Gaussian Linear State-Space Models" (CGLSSM), considered as a natural way to extend Gaussian systems to Gaussian switching ones, do not allow for a filtering scheme that can be performed in a reasonable running time [1]-[5]. Classically, CGLSSMs rely on the following assumptions:

1) $\boldsymbol{R}_{1}^{N}$ is Markov;

2) $\boldsymbol{X}_{1}^{N}$ is Markov conditionally on $\boldsymbol{R}_{1}^{N}$;

3) $\left(Y_{n}\right), 1 \leq n \leq N$, are Gaussian, independent conditionally on $\left(\boldsymbol{R}_{1}^{N}, \boldsymbol{X}_{1}^{N}\right)$ and verify:

$$
p\left(y_{n} \mid \boldsymbol{r}_{1}^{N}, \boldsymbol{x}_{1}^{N}\right)=p\left(y_{n} \mid r_{n}, x_{n}\right) .
$$

In CGLSSMs, $\boldsymbol{R}_{1}^{N}$ and $\left(\boldsymbol{R}_{1}^{N}, \boldsymbol{X}_{1}^{N}\right)$ are both Markov, and $p\left(y_{n} \mid \boldsymbol{r}_{1}^{N}, \boldsymbol{x}_{1}^{N}\right)$ is very simple. These assumptions do not allow for exact computation of recursive filters, since $\left(\boldsymbol{R}_{1}^{N}, \boldsymbol{Y}_{1}^{N}\right)$ is not Markov and $p\left(r_{n} \mid \boldsymbol{y}_{1}^{n}\right)$ cannot be computed sequentially and exactly. This problem has been addressed in recent "conditionally Markov switching hidden linear models" (CMSHLMs [6]), in which both $\boldsymbol{R}_{1}^{N}$ and $\left(\boldsymbol{R}_{1}^{N}, \boldsymbol{Y}_{1}^{N}\right)$ are Markov, and $p\left(x_{n+1} \mid \boldsymbol{r}_{1}^{n}, \boldsymbol{x}_{1}^{n}\right)$ is pretty general. Here we consider particular Gaussian CMSHLMs called "Conditionally Gaussian Observed Markov Switching Models" (CGOMSMs [7][9]), which verify:

1) $\boldsymbol{R}_{1}^{N},\left(\boldsymbol{R}_{1}^{N}, \boldsymbol{Y}_{1}^{N}\right)$ and $\left(\boldsymbol{X}_{1}^{N}, \boldsymbol{R}_{1}^{N}, \boldsymbol{Y}_{1}^{N}\right)$ are Markov;

2) $\left(\boldsymbol{X}_{1}^{N}, \boldsymbol{Y}_{1}^{N}\right)$ is Gaussian conditionally on $\boldsymbol{R}_{1}^{N}$.

Figure 1 illustrates the dependencies between the stochastic processes defining the studied CGOMSM. Such an approach is different from classic ones since the hidden chain $\left(\boldsymbol{R}_{1}^{N}, \boldsymbol{X}_{1}^{N}\right)$ is no longer assumed 
Markov, as it has been usually done. Thus, recursive exact filtering is feasible in CGOMSMs and the interesting point is that these models can be quite close to the classic CGLSSMs [7], [10].

The values $\boldsymbol{R}_{n}^{n+1}=\boldsymbol{r}_{n}^{n+1}$ govern the parameters of the distribution

$$
p\left(x_{n+1}, y_{n+1} \mid x_{n}, \boldsymbol{r}_{n}^{n+1}, y_{n}\right)
$$

and thus there exist four possible transitions according to $\boldsymbol{r}_{n}^{n+1} \in$ $5 \Omega^{2}=\{0,1\}^{2}$. The main limitation of the classical switching model which relies on hard switches is that it does not take into account the transient transition between switches. In real world applications (c.f. Section III), the crisp transition can cause the model to discard significant information that corresponds to the time period during

10 which the system switches from one regime to another. Therefore, the hard switches modeling impacts the accuracy of the filtering scheme. If we consider the example of tracking a moving object using sensors in intermittent sunlight and shadow conditions (both corresponding to hard switches 0 and 1), there exist intermediary situations as

15 sunlight condition may fade into shadow in a continuous manner. Considering only the hard switches imposes the filtering method to consider only one of the states 0 and 1 during the transition and consequently this shall compromise the accuracy of the filtering since the system state during the transitory phase is a mixture of the two

20 hard switches. Thus, a more complete model would be to associate a set of parameters to each $\boldsymbol{r}_{n}^{n+1} \in[0,1]^{2}$. This is the very aim of the paper: we extend the Markov chain $\boldsymbol{R}_{1}^{N}$ used in CGOMSMs to a 'fuzzy' one. Such models called 'fuzzy models', have been proposed in [11] in a simple context, without Markovianity, to deal with fuzzy image segmentation. Then, they have been extensively used in hidden Markov chains [12]-[14] and hidden Markov fields [14]-[16]. Here, the hidden fuzzy Markov chain will be considered to model the pair (switches, observations) in the context of filtering in presence of jumps, which is possible as the pair $\left(\boldsymbol{R}_{1}^{N}, \boldsymbol{Y}_{1}^{N}\right)$ is Markov in 30 CGOMSMs.

To the best of our knowledge, while there exist several research works dealing with fuzzy Markov models, the literature for fuzzy Markov jump model filtering is surprisingly scant despite its practical potential. Recently, the discrete-time Takagi-Sugano (T.-S.) approach

35 to fuzzy filter design for Markovian jump model has been gaining a significant interest over the last few years -see for example [17], [18] and the references therein- especially in the fuzzy control and fault detection research community. Unlike T.-S. model, our approach assumes that, conditionally to jumps, the model is pairwise 40 linear, which enriches the classical linear models. The proposed fuzzy filter allows for exact calculations for the filter, up to numerical approximation of some integrals, as precised in the text. In the remaining, we start with a brief description of the CGOMSM in Section II] and pursue with the description of the original fuzzy jump

45 model in Section III Sections IV and $\mathrm{V}$ depict how the corresponding 'fuzzy' filter runs. Section VI reports experimental results that show how the fuzzy filter can improve filtering from the CGOMSM, while Section VII reports comparative filtering result on real data.

\section{Conditionally Gaussian observed Markov hard} SWITCHING MODEL

Let us set $\boldsymbol{Z}_{n}=\left(X_{n}, Y_{n}\right)^{\top}, \boldsymbol{T}_{n}=\left(X_{n}, R_{n}, Y_{n}\right)^{\boldsymbol{\top}}$ and assume the following:

1) $\boldsymbol{T}_{1}^{N}$ is Markov;

2) $p\left(r_{n+1} \mid \boldsymbol{t}_{n}\right)=p\left(r_{n+1} \mid r_{n}\right)$, which implies the Markovianity of $\boldsymbol{R}_{1}^{N}$

3) $\boldsymbol{Z}_{1}^{N}=\left(\boldsymbol{Z}_{1}, \ldots, \boldsymbol{Z}_{N}\right)$ is Gaussian conditionally on $\boldsymbol{R}_{1}^{N}$.

Such a model, introduced in [8], is called "Conditionally Gaussian Markov Switching Model" (CGMSM), and is defined by $p\left(t_{1}\right)$, transitions $p\left(r_{n+1} \mid r_{n}\right)$, and

$$
\begin{array}{r}
\boldsymbol{Z}_{n+1}=\boldsymbol{A}_{n+1}\left(\boldsymbol{r}_{n}^{n+1}\right) \boldsymbol{Z}_{n}+\boldsymbol{B}_{n+1}\left(\boldsymbol{r}_{n}^{n+1}\right) \boldsymbol{W}_{n+1} \\
+\boldsymbol{N}_{n+1}\left(\boldsymbol{r}_{n}^{n+1}\right),
\end{array}
$$

for $n=1, \ldots, N-1$, and where

- $\boldsymbol{W}_{n}=\left(U_{n}, V_{n}\right)^{\top}$ with $U_{1}, V_{1}, \ldots, U_{N}, V_{N}$ Gaussian zeromean independent vectors with identity covariance matrices;

- Matrices $\boldsymbol{A}_{n+1}\left(\boldsymbol{r}_{n}^{n+1}\right)$ and $\boldsymbol{B}_{n+1}\left(\boldsymbol{r}_{n}^{n+1}\right)$ :

$$
\begin{aligned}
& \boldsymbol{A}_{n+1}\left(\boldsymbol{r}_{n}^{n+1}\right)=\left[\begin{array}{ll}
a_{n+1}^{1}\left(\boldsymbol{r}_{n}^{n+1}\right) & a_{n+1}^{2}\left(\boldsymbol{r}_{n}^{n+1}\right) \\
a_{n+1}^{3}\left(\boldsymbol{r}_{n}^{n+1}\right) & a_{n+1}^{4}\left(\boldsymbol{r}_{n}^{n+1}\right)
\end{array}\right], \\
& \boldsymbol{B}_{n+1}\left(\boldsymbol{r}_{n}^{n+1}\right)=\left[\begin{array}{ll}
b_{n+1}^{1}\left(\boldsymbol{r}_{n}^{n+1}\right) & b_{n+1}^{2}\left(\boldsymbol{r}_{n}^{n+1}\right) \\
b_{n+1}^{3}\left(\boldsymbol{r}_{n}^{n+1}\right) & b_{n+1}^{4}\left(\boldsymbol{r}_{n}^{n+1}\right)
\end{array}\right] ;
\end{aligned}
$$

- Means $\boldsymbol{N}_{n+1}\left(\boldsymbol{r}_{n}^{n+1}\right)=\left(N_{n+1}^{X}\left(\boldsymbol{r}_{n}^{n+1}\right), N_{n+1}^{Y}\left(\boldsymbol{r}_{n}^{n+1}\right)\right)^{\top}$ are given by

$$
\boldsymbol{N}_{n+1}\left(\boldsymbol{r}_{n}^{n+1}\right)=\boldsymbol{M}_{n+1}\left(r_{n+1}\right)-\boldsymbol{A}_{n+1}\left(\boldsymbol{r}_{n}^{n+1}\right) \boldsymbol{M}_{n}\left(r_{n}\right),
$$

with

$$
\boldsymbol{M}_{n}\left(r_{n}\right)=\mathbb{E}\left[\left(\begin{array}{c}
X_{n} \\
Y_{n}
\end{array}\right) \mid r_{n}\right]=\left[\begin{array}{l}
M_{n}^{X}\left(r_{n}\right) \\
M_{n}^{Y}\left(r_{n}\right)
\end{array}\right] .
$$

Recursive filtering is not workable in the general CGMSM. Besides, let us notice that the classic "Conditionally Gaussian linear state-space model" (CGLSSM [2]) is a particular CGMSM obtained by setting, for each $\boldsymbol{r}_{n}^{n+1} \in \Omega^{2}$

$$
a_{n+1}^{2}\left(\boldsymbol{r}_{n}^{n+1}\right)=a_{n+1}^{4}\left(\boldsymbol{r}_{n}^{n+1}\right)=b_{n+1}^{2}\left(\boldsymbol{r}_{n}^{n+1}\right)=0 .
$$

However, another particular CGMSM, called "Conditionally Gaussian observed Markov switching model" (CGOMSM) obtained from CGMSM by setting, for each $\boldsymbol{r}_{n}^{n+1} \in \Omega^{2}[7 \mid-[10]$

$$
a_{n+1}^{3}\left(\boldsymbol{r}_{n}^{n+1}\right)=0,
$$

allows for recursive optimal filtering even with switches [8]. Indeed, CGOMSM belongs to the category of "conditionally Markov switching hidden linear models" (CMSHLMs) in which recursive optimal filtering is workable [6].

The aim of this paper is to extend the CGOMSM defined by (1)(3) and eq. (5) to a 'fuzzy' CGOMSM (denoted by CGOFMSM) and to show how the related recursive optimal 'fuzzy' filter runs.

\section{Conditionally GaUsSian OBSERVED fUZZY MARKov SWITCHING MODEL}

Let us begin by illustrating with three examples the interest of the new proposed model in real situations.

In the first example, let sequence $\boldsymbol{X}_{1}^{N}$ model the positions at time index $1, \ldots, N$ of a flying object, and let sequence $\boldsymbol{Y}_{1}^{N}$ model the measurements provided by some optical sensors situated on the ground. During the tracking process, the sunlight can be partially or totally hidden due to the presence of clouds, which gives two models for the distribution of $\boldsymbol{Y}_{1}^{N}$. This can be modelled by a 'hard' model with $\boldsymbol{R}_{1}^{N}$ such that each $R_{n}$ takes its value in $\Omega=\{0,1\}$, with 0 corresponds to total sunlight exposure and 1 to shadow condition. In some situations, during cloudy weather conditions that hide the sun partially, the transition from sunlight to shadow is 'continuous', and the duration of 'intermediary' light can be of paramount importance to the tracking process. This motivates the introduction of 'fuzzy' model with each $R_{n}$ belonging to $\Omega=[0,1]$ rather than to $\Omega=\{0,1\}$.

However, the distribution of $R_{n}$ on $\Omega=[0,1]$ has to verify some properties. Willing to have non-null probability to have sunshine and likewise for shadow - implies that there should be two Dirac masses on 0 and 1 . Then one can complete the distribution of $R_{n}$ 
on $\Omega=[0,1]$ by setting continuous probability on $] 0,1[$. Finally, the distribution of $R_{n}$ is defined by its density $p:[0,1] \rightarrow \mathbb{R}$ with respect to $\nu=\delta_{0}+\delta_{1}+\mu_{0,1}$, where $\delta_{0}, \delta_{1}$ are Dirac's distributions on 0,1 , and $\mu_{0,1[}$ is the Lebesgue's measure on $] 0,1[$.

Let us consider a second example dealing with pedestrian tracking for surveillance purposes which consists in tracking the movements of pedestrians using aggregated data acquired from deployed sensors in the monitored area [19]. Due to the dynamic aspect of pedestrian motion in the presence of several contextual information such as

10 crowd, the use of a two-motion model (corresponding to crowded / uncrowned configurations) is necessary. However, the concept of 'crowd' can be seen as a fuzzy phenomenon. Hence, relying on an abrupt change of parameters within the two-jump scheme does not take into account the intermediate states of pedestrian motion 15 and impacts the accuracy of tracking process. Another example of the same problem relates to car traffic speed and density in a road segment [20].

A last example showing the potential interest of a fuzzy model appears when we want to study the phenomenon associated to out-

20 door air temperature. Typically, during one day, temperature reaches minimal values during the night and maximal during the afternoon. Between these two ranges, temperatures increase and decrease and can be represented by the fuzzy nature of the jumps considered in our model. An example of such a situation is detailed in Section VII

From this perspective, the use of fuzzy transitions to model the transient change of parameters is more relevant than the salient switching model. The definition of the new "Conditionally Gaussian Observed Fuzzy Markov Switching Model" (CGOFMSM) we propose is similar to (1)-(3) and eq. (5), except that we limit our study 30 to two hard classes and each $R_{n}$ takes its values in $\Omega=[0,1]$.

Definition 1. Let $\boldsymbol{X}_{1}^{N}, \boldsymbol{Y}_{1}^{N}$ and $\boldsymbol{R}_{1}^{N}$ be three stochastic sequences of random variables taking their values in $\mathbb{R}, \mathbb{R}$ and $[0,1]$ respectively. The triplet $\boldsymbol{T}_{1}^{N}=\left(\boldsymbol{T}_{1}, \ldots, \boldsymbol{T}_{N}\right)$, with $\boldsymbol{T}_{n}=\left(X_{n}, R_{n}, Y_{n}\right)^{\top}$, will be said "Conditionally Gaussian observed fuzzy Markov switching

1) $\boldsymbol{T}_{1}^{N}$ verifies (1)-(3) and eq. (5);

2) The distribution of each $R_{n}$ is defined by a density (possibly depending on n) $p:[0,1] \rightarrow \mathbb{R}$ with respect to $\nu=\delta_{0}+\delta_{1}+$ $\mu_{0,1}\left[\right.$, where $\delta_{0}, \delta_{1}$ are Dirac's distributions on 0,1 , and $\mu_{0,1}[$ is the Lebesgue's measure on ]0, 1 .

Let us recall some basic rules for integrating a function with respect to $\nu=\delta_{0}+\delta_{1}+\mu_{0,1}$. Such integration has two components: sum of its values on 0,1 , and 'classic' integration over ] 0,1 [. More precisely, for any function $\phi:[0,1] \rightarrow \mathbb{R}$, we have

$$
\begin{aligned}
\int_{0}^{1} \phi(r) d \nu(r) & =\int_{0}^{1} \phi(r)\left(\delta_{0}+\delta_{1}+\mu_{0,1}\right) \\
& =\phi(0)+\phi(1)+\int_{0}^{1} \phi(r) d r .
\end{aligned}
$$

45 In particular, the expectation of $\phi\left(R_{n}\right)$ is written

$$
\begin{aligned}
\mathbb{E}\left[\phi\left(r_{n}\right)\right] & =\int_{0}^{1} \phi(r) p(r) d \nu(r)=\phi(0) p(0) \\
& +\phi(1) p(1)+\int_{0}^{1} \phi(r) p(r) d r .
\end{aligned}
$$

The distribution of a fuzzy Markov chain (FMC) $\boldsymbol{R}_{1}^{N}$ is defined by the density $p\left(r_{1}\right)$ and the conditional densities $p\left(r_{n+1} \mid r_{n}\right)$. All of them are thus defined on $\Omega=[0,1]$ and are densities w.r.t. $\nu=$ $\delta_{0}+\delta_{1}+\mu_{0,1}$. According to the general integration w.r.t. $\nu$ rule in eq. [6, we have

$$
\int_{0}^{1} p\left(r_{1}\right) d \nu\left(r_{1}\right)=p(0)+p(1)+\int_{0}^{1} p\left(r_{1}\right) d r_{1}=1
$$

and

$$
\begin{aligned}
p\left(r_{n+1}\right)= & \int_{0}^{1} p\left(r_{n}\right) p\left(r_{n+1} \mid r_{n}\right) d \nu\left(r_{n}\right) \\
= & p(0) p\left(r_{n+1} \mid 0\right)+p(1) p\left(r_{n+1} \mid 1\right) \\
& \quad+\int_{0}^{1} p\left(r_{n}\right) p\left(r_{n+1} \mid r_{n}\right) d r_{n} .
\end{aligned}
$$

Finally, optimal filtering in 'fuzzy' CGOFMSM is not very different from that in 'hard' CGOMSM, the difference being that, in CGOMSM, integrating with respect to $r_{n}$ consists in summing, while in CGOFMSM it consists of integrating with respect to $\nu$.

\section{OPTIMAL FUZZY SWITCHING RECURSIVE FILTER}

We wish to compute $p\left(r_{n+1} \mid \boldsymbol{y}_{1}^{n+1}\right), \mathbb{E}\left[X_{n+1} \mid r_{n+1}, \boldsymbol{y}_{1}^{n+1}\right]$, and $\mathbb{E}\left[X_{n+1}^{2} \mid r_{n+1}, \boldsymbol{y}_{1}^{n+1}\right]$ from $p\left(r_{n} \mid \boldsymbol{y}_{1}^{n}\right), \mathbb{E}\left[X_{n} \mid r_{n}, \boldsymbol{y}_{1}^{n}\right]$, $\mathbb{E}\left[X_{n}^{2} \mid r_{n}, \boldsymbol{y}_{1}^{n}\right]$, and $y_{n+1}$. According to eq. (2) and (5), $\left(\boldsymbol{R}_{1}^{N}, \boldsymbol{Y}_{1}^{N}\right)$ is a hidden Markov chain, which makes possible the computation of $p\left(r_{n+1} \mid \boldsymbol{y}_{1}^{n+1}\right)$ as explained below.

First, let us note that the probabilities

$p\left(\boldsymbol{r}_{n}^{n+1}, y_{n+1} \mid \boldsymbol{y}_{1}^{n}\right)=p\left(y_{n+1} \mid \boldsymbol{r}_{n}^{n+1}, y_{n}\right) p\left(r_{n} \mid \boldsymbol{y}_{1}^{n}\right) p\left(r_{n+1} \mid r_{n}\right)$,

can be calculated since

- $p\left(r_{n+1} \mid r_{n}\right)$ are given;

- $p\left(r_{n} \mid \boldsymbol{y}_{1}^{n}\right)$ can be calculated from(13);

- $p\left(y_{n+1} \mid \boldsymbol{r}_{n}^{n+1}, y_{n}\right)$ are conditional densities of the multivariate Gaussians defined by (2). Taking into account (5), by using [21 Section 8.1.3, page 40], we obtain means and variances of $a_{n+1}^{4}\left(\boldsymbol{r}_{n}^{n+1}\right)\left(y_{n}-M_{n}^{Y}\left(r_{n}\right)\right)+M_{n+1}^{Y}\left(r_{n+1}\right)$ and $\left(b_{n+1}^{3}\left(\boldsymbol{r}_{n}^{n+1}\right)\right)^{2}+\left(b_{n+1}^{4}\left(\boldsymbol{r}_{n}^{n+1}\right)\right)^{2}$ respectively.

Secondly, the following probabilities

$$
p\left(y_{n+1} \mid \boldsymbol{y}_{1}^{n}\right)=\iint_{0}^{1} p\left(\boldsymbol{r}_{n}^{n+1}, y_{n+1} \mid \boldsymbol{y}_{1}^{n}\right)\left(d \nu\left(r_{n}\right) \otimes d \nu\left(r_{n+1}\right)\right),
$$

can also be computed accordingly:

$$
p\left(\boldsymbol{r}_{n}^{n+1} \mid \boldsymbol{y}_{1}^{n+1}\right)=\frac{p\left(\boldsymbol{r}_{n}^{n+1}, y_{n+1} \mid \boldsymbol{y}_{1}^{n}\right)}{p\left(y_{n+1} \mid \boldsymbol{y}_{1}^{n}\right)} .
$$

Finally, using (10), eq. (12) gives the so-called forward probabilities

$$
\begin{aligned}
p\left(r_{n+1} \mid \boldsymbol{y}_{1}^{n+1}\right)= & \int_{0}^{1} p\left(\boldsymbol{r}_{n}^{n+1} \mid \boldsymbol{y}_{1}^{n+1}\right) d \nu\left(r_{n}\right) \\
& \frac{\int_{0}^{1} p\left(\boldsymbol{r}_{n}^{n+1}, y_{n+1} \mid \boldsymbol{y}_{1}^{n}\right) d \nu\left(r_{n}\right)}{p\left(y_{n+1} \mid \boldsymbol{y}_{1}^{n}\right)} .
\end{aligned}
$$

Also, for the later use, note that

$$
p\left(r_{n} \mid r_{n+1}, \boldsymbol{y}_{1}^{n+1}\right)=\frac{p\left(\boldsymbol{r}_{n}^{n+1} \mid \boldsymbol{y}_{1}^{n+1}\right)}{p\left(r_{n+1} \mid \boldsymbol{y}_{1}^{n+1}\right)} .
$$

The "optimal fuzzy switching recursive filter" (OFSRF) we propose consists of five steps outlined as follows. To start the iterations, we first use the distribution of $\boldsymbol{T}_{1}$. It is then possible to run the OFSRF iterations, assuming that all quantities have been computed for sample $n$ :

1) Compute $p\left(r_{n+1} \mid \boldsymbol{y}_{1}^{n+1}\right)$ with 12)-13.

2) Compute $\mathbb{E}\left[\boldsymbol{Z}_{n+1} \mid \boldsymbol{r}_{n}^{n+1}, \boldsymbol{y}_{1}^{n}\right]$ and $\operatorname{Var}\left[\boldsymbol{Z}_{n+1} \mid \boldsymbol{r}_{n}^{n+1}, \boldsymbol{y}_{1}^{n}\right]$. From (2), we have

$$
\begin{gathered}
\mathbb{E}\left[\boldsymbol{Z}_{n+1} \mid \boldsymbol{r}_{n}^{n+1}, \boldsymbol{y}_{1}^{n}\right] \\
=\boldsymbol{A}_{n+1}\left(\boldsymbol{r}_{n}^{n+1}\right) \mathbb{E}\left[\boldsymbol{Z}_{n} \mid \boldsymbol{r}_{n}^{n+1}, \boldsymbol{y}_{1}^{n}\right]+\boldsymbol{N}_{n+1}\left(\boldsymbol{r}_{n}^{n+1}\right),
\end{gathered}
$$


Recalling that $R_{n+1}$ and $\boldsymbol{Z}_{n}$ are independent conditionally on $R_{n}$ (Condition 2 in the definition of CGMSM), we have

$$
\mathbb{E}\left[\boldsymbol{Z}_{n} \mid \boldsymbol{r}_{n}^{n+1}, \boldsymbol{y}_{1}^{n}\right]=\left[\begin{array}{c}
\left.\mathbb{E}\left[X_{n} \mid r_{n}, \boldsymbol{y}_{1}^{n}\right]\right] \\
y_{n}
\end{array}\right]
$$

Also, using (2) and from classical calculations detailed in C. we have

$$
\begin{gathered}
\operatorname{Var}\left[\boldsymbol{Z}_{n+1} \mid \boldsymbol{r}_{n}^{n+1}, \boldsymbol{y}_{1}^{n}\right] \\
=\boldsymbol{B}_{n+1}\left(\boldsymbol{r}_{n}^{n+1}\right) \boldsymbol{B}_{n+1}^{\top}\left(\boldsymbol{r}_{n}^{n+1}\right) \\
+\boldsymbol{A}_{n+1}\left(\boldsymbol{r}_{n}^{n+1}\right) \operatorname{Var}\left[\boldsymbol{Z}_{n} \mid \boldsymbol{r}_{n}^{n+1}, \boldsymbol{y}_{1}^{n}\right] \boldsymbol{A}_{n+1}^{\top}\left(\boldsymbol{r}_{n}^{n+1}\right) \\
=\boldsymbol{B}_{n+1}\left(\boldsymbol{r}_{n}^{n+1}\right) \boldsymbol{B}_{n+1}^{\top}\left(\boldsymbol{r}_{n}^{n+1}\right) \\
+\boldsymbol{A}_{n+1}\left(\boldsymbol{r}_{n}^{n+1}\right) \operatorname{Var}\left[\boldsymbol{Z}_{n} \mid r_{n}, \boldsymbol{y}_{1}^{n}\right] \boldsymbol{A}_{n+1}^{\top}\left(\boldsymbol{r}_{n}^{n+1}\right) .
\end{gathered}
$$

For the later convenience, let us note:

$$
\operatorname{Var}\left[\boldsymbol{Z}_{n+1} \mid \boldsymbol{r}_{n}^{n+1}, \boldsymbol{y}_{1}^{n}\right]=\left[\begin{array}{ll}
\alpha_{n+1}\left(\boldsymbol{r}_{n}^{n+1}\right) & \beta_{n+1}\left(\boldsymbol{r}_{n}^{n+1}\right) \\
\xi_{n+1}\left(\boldsymbol{r}_{n}^{n+1}\right) & \delta_{n+1}\left(\boldsymbol{r}_{n}^{n+1}\right)
\end{array}\right]
$$

3) From the multivariate normal distribution specified by 15 and (16, compute the parameters $\mathbb{E}\left[X_{n+1} \mid \boldsymbol{r}_{n}^{n+1}, \boldsymbol{y}_{1}^{n+1}\right.$ and $\mathbb{E}\left[X_{n+1}^{2} \mid \boldsymbol{r}_{n}^{n+1}, \boldsymbol{y}_{1}^{n+1}\right]$ of its marginal $X_{n+1} \mid y_{n+1}, \boldsymbol{r}_{n}^{n+1}$ (see [21. Section 8.1.3, page 40]) according to

$$
\begin{gathered}
\mathbb{E}\left[X_{n+1} \mid \boldsymbol{r}_{n}^{n+1}, \boldsymbol{y}_{1}^{n+1}\right] \\
=\mathbb{E}\left[X_{n+1} \mid \boldsymbol{r}_{n}^{n+1}, \boldsymbol{y}_{1}^{n}\right]+\frac{\beta_{n+1}\left(\boldsymbol{r}_{n}^{n+1}\right)}{\delta_{n+1}\left(\boldsymbol{r}_{n}^{n+1}\right)} \\
\left(y_{n+1}-\mathbb{E}\left[Y_{n+1} \mid \boldsymbol{r}_{n}^{n+1}, \boldsymbol{y}_{1}^{n}\right]\right),
\end{gathered}
$$

with $\mathbb{E}\left[X_{n+1} \mid \boldsymbol{r}_{n}^{n+1}, \boldsymbol{y}_{1}^{n}\right]$ and $\mathbb{E}\left[Y_{n+1} \mid \boldsymbol{r}_{n}^{n+1}, \boldsymbol{y}_{1}^{n}\right]$ given by eq. [15, and

$$
\begin{gathered}
\mathbb{E}\left[X_{n+1}^{2} \mid \boldsymbol{r}_{n}^{n+1}, \boldsymbol{y}_{1}^{n+1}\right] \\
=\mathbb{E}^{2}\left[X_{n+1} \mid \boldsymbol{r}_{n}^{n+1}, \boldsymbol{y}_{1}^{n+1}\right]+\alpha_{n+1}\left(\boldsymbol{r}_{n}^{n+1}\right) \\
-\frac{\beta_{n+1}\left(\boldsymbol{r}_{n}^{n+1}\right)}{\delta_{n+1}\left(\boldsymbol{r}_{n}^{n+1}\right)} \xi_{n+1}\left(\boldsymbol{r}_{n}^{n+1}\right) ;
\end{gathered}
$$

4) Compute $\mathbb{E}\left[X_{n+1} \mid r_{n+1}, \boldsymbol{y}_{1}^{n+1}\right]$ and $\mathbb{E}\left[X_{n+1}^{2} \mid r_{n+1}, \boldsymbol{y}_{1}^{n+1}\right]$ using (14) with

$$
\begin{gathered}
\mathbb{E}\left[X_{n+1} \mid r_{n+1}, \boldsymbol{y}_{1}^{n+1}\right] \\
\left.=\int_{0}^{1} \mathbb{E}\left[X_{n+1} \mid \boldsymbol{r}_{n}^{n+1}, \boldsymbol{y}_{1}^{n+1}\right] p\left(r_{n} \mid r_{n+1}, \boldsymbol{y}_{1}^{n+1}\right) d \nu\left(r_{n}\right) 19\right) \\
\mathbb{E}\left[X_{n+1}^{2} \mid r_{n+1}, \boldsymbol{y}_{1}^{n+1}\right] \\
=\int_{0}^{1} \mathbb{E}\left[X_{n+1}^{2} \mid \boldsymbol{r}_{n}^{n+1}, \boldsymbol{y}_{1}^{n+1}\right] p\left(r_{n} \mid r_{n+1}, \boldsymbol{y}_{1}^{n+1}\right) d \nu\left(r_{n}\right)(20)
\end{gathered}
$$

5) Finally, compute the filtering equations

$$
\begin{gathered}
\mathbb{E}\left[X_{n+1} \mid \boldsymbol{y}_{1}^{n+1}\right] \\
=\int_{0}^{1} \mathbb{E}\left[X_{n+1} \mid r_{n+1}, \boldsymbol{y}_{1}^{n+1}\right] p\left(r_{n+1} \mid \boldsymbol{y}_{1}^{n+1}\right) d \nu\left(r_{n+1}\right)(21)
\end{gathered}
$$

and

$$
\begin{gathered}
\mathbb{E}\left[X_{n+1}^{2} \mid \boldsymbol{y}_{1}^{n+1}\right] \\
=\int_{0}^{1} \mathbb{E}\left[X_{n+1}^{2} \mid r_{n+1}, \boldsymbol{y}_{1}^{n+1}\right] p\left(r_{n+1} \mid \boldsymbol{y}_{1}^{n+1}\right) d \nu\left(r_{n+1}\right)(22)
\end{gathered}
$$

Remark 1. Integration with respect to $\nu$ above cannot be written in a closed-form formula. It is, then, approximated by numerical integration. Let $F$ denote the number of discrete steps used to compute integrals on $] 0,1[$. The impact of $F$ on the restoration results

\section{MODEL PARAMETRIZATION}

In order to assess the interest of the filtering algorithm on CGOFMSM simulated data, we will consider stationary CGOFMSM models, with distribution defined by $p\left(\boldsymbol{x}_{1}^{2}, \boldsymbol{r}_{1}^{2}, \boldsymbol{y}_{1}^{2}\right)=$ $p\left(\boldsymbol{r}_{1}^{2}\right) p\left(\boldsymbol{x}_{1}^{2}, \boldsymbol{y}_{1}^{2} \mid \boldsymbol{r}_{1}^{2}\right)$. Thus, we have to define $p\left(\boldsymbol{r}_{1}^{2}\right)$ (Section $\mathrm{V}$-A and $p\left(\boldsymbol{x}_{1}^{2}, \boldsymbol{y}_{1}^{2} \mid \boldsymbol{r}_{1}^{2}\right)$ (Section V-B. Thanks to the particular structure of $\nu$, this can be done in such a way that when the fuzziness disappears, it is to say when $p\left(r_{n}\right)=0$ on $] 0,1[$ for $n=1, \ldots, N$, a CGOFMSM becomes a classical CGOMSM.

\section{A. Distribution of $\left(R_{1}, R_{2}\right)$}

Let us notice that in the 'hard' case with two possible switches, the distribution $P_{\left(R_{1}, R_{2}\right)}$ is simply a probability over $\{0,1\}^{2}$. In the fuzzy case we deal with, it is a distribution on $[0,1]^{2}$, which provides a wide range of possibilities for choosing its shape. We next describe two possible shapes of interest for $P_{\left(R_{1}, R_{2}\right)}$ (called FMC1 and FMC2 models, where FMC stands for 'Fuzzy Markov Chain'), that will be experimented in next Section.

1) First case (FMC1 model): The density $p\left(\boldsymbol{r}_{1}^{2}\right)$ of $P_{\left(\boldsymbol{R}_{1}^{2}\right)}$ w.r.t. $\nu \otimes \nu$ - where $\nu=\delta_{0}+\delta_{1}+\mu_{0,1[}$ - is of the form:

$$
\begin{aligned}
& p(0,0)=p(1,1)=\alpha, \\
& p(1,0)=p(0,1)=\beta, \\
& \begin{aligned}
p\left(r_{1}, r_{2}\right)=\eta+ & (\delta-\eta)\left|r_{1}-r_{2}\right|, \\
& \quad \text { for }\left(r_{1}, r_{2}\right) \in[0,1]^{2} \backslash\{0,1\}^{2},
\end{aligned}
\end{aligned}
$$

$\iint_{0}^{1} p\left(r_{1}, r_{2}\right) d \nu\left(r_{1}\right) d \nu\left(r_{2}\right)=1$. A possible shape for this density is illustrated in Fig. 2

Remark 2. We obtain a 'fuzzy constant' model by setting $\delta=\eta$ and, in particular, we get a 'purely hard' CGOMSM model by setting $\delta=\eta=0$.

The density $p\left(r_{1}\right)$ of $P_{\left(R_{1}\right)}$ is computed as follows:

$$
p\left(r_{1}\right)= \begin{cases}\alpha+\beta+\frac{\delta+\eta}{2} & \text { if } r_{1}=0, \\ \alpha+\beta+\frac{\delta+\eta}{2} & \text { if } r_{1}=1, \\ \frac{3}{2}(\delta+\eta)+(\delta-\eta)\left(r_{1}^{2}-r_{1}\right) & \text { if } \left.r_{1} \in\right] 0,1[.\end{cases}
$$

Knowing that $\int_{0}^{1} p\left(r_{1}\right) d \nu\left(r_{1}\right)=1$, we get

$$
\beta=\frac{1-\frac{5}{2}(\delta+\eta)+\frac{1}{6}(\delta-\eta)}{2}-\alpha \text {. }
$$

Hence, this model is only parametrized by $\{\alpha, \delta, \eta\}$ (the calculations are detailed in Appendix A].

The limit proportion of hard data $\left(p_{H}\right)$ with respect to fuzzy ones $\left(p_{F}\right)$ in a sampled sequence is

$$
\begin{aligned}
& p_{H}=p(0)+p(1)=2(\alpha+\beta)+(\delta+\eta), \\
& p_{F}=1-p_{H}=\frac{3}{2}(\delta+\eta)-\frac{1}{6}(\delta-\eta) .
\end{aligned}
$$

The density $p\left(r_{2} \mid r_{1}\right)$ of distribution $P_{R_{2} \mid R_{1}}$, w.r.t. $\nu$, is the ratio between the joint density and the marginal density. We have to distinguish between different cases, according to the value of $r_{1}$ :

$$
\begin{aligned}
& p\left(r_{2} \mid r_{1}=0\right)= \begin{cases}\frac{\alpha}{D_{1}} & \text { if } r_{2}=0, \\
\frac{\beta}{D_{1}} & \text { if } r_{2}=1, \\
\frac{\eta+(\delta-\eta) r_{2}}{D_{1}} & \text { if } \left.r_{2} \in\right] 0,1[.\end{cases} \\
& p\left(r_{2} \mid r_{1}=1\right)= \begin{cases}\frac{\beta}{D_{1}} & \text { if } r_{2}=0, \\
\frac{\alpha}{D_{1}} & \text { if } r_{2}=1, \\
\frac{\delta+(\eta-\delta) r_{2}}{D_{1}} & \text { if } \left.r_{2} \in\right] 0,1[.\end{cases} \\
& p\left(r_{2} \mid r_{1} \in\right] 0,1[)= \begin{cases}\frac{\eta+(\delta-\eta) r_{1}}{D_{2}} & \text { if } r_{2}=0, \\
\frac{\delta+(\eta-\delta) r_{1}}{D_{2}} & \text { if } r_{2}=1, \\
\frac{\eta+(\delta-\eta)\left|r_{1}-r_{2}\right|}{D_{2}} & \text { if } \left.r_{2} \in\right] 0,1[.\end{cases}
\end{aligned}
$$




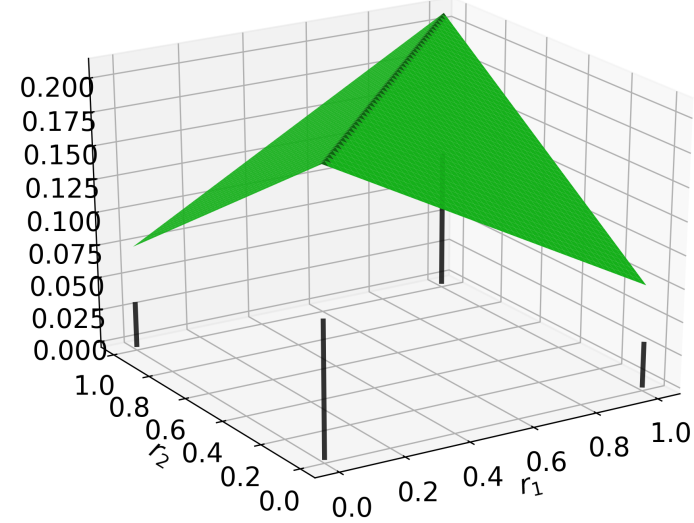

(a) Parameters: $\alpha=0.10, \eta=0.21, \delta=0.076\left(\beta=0.03, p_{H}=\right.$ $0.55)$.

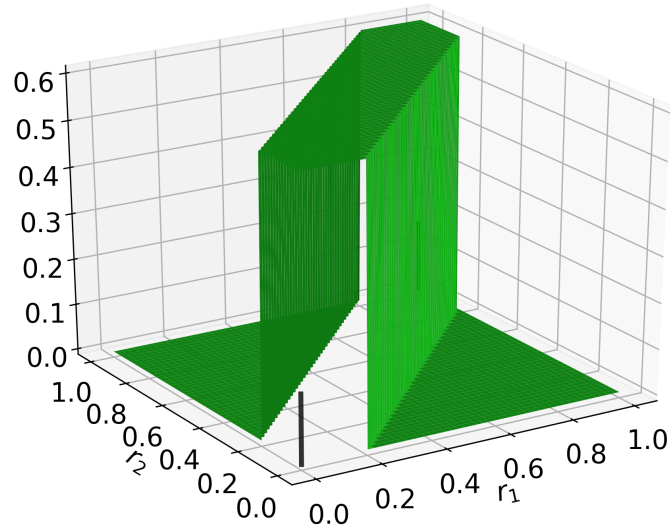

(b) Parameters: $\alpha=0.15, \gamma=0.60, \delta=0.20\left(\beta=0.0, p_{H}=\right.$ $0.54)$.

Fig. 2. Density $p\left(r_{1}, r_{2}\right)$ for the FMC1 model (a) and for the FMC2 model (b).

with $D_{1}=\alpha+\beta+\frac{\delta+\eta}{2}$ and $D_{2}=\frac{3}{2}(\delta+\eta)+(\delta-\eta)\left(r_{1}^{2}-r_{1}\right)$.

For the particular case where $\delta=\eta=0$, we have a classical Markov chain with 2 states, and $p\left(r_{2} \mid r_{1}\right)$ writes

$$
\begin{aligned}
& p\left(r_{2} \mid r_{1}=0\right)= \begin{cases}\frac{\alpha}{\alpha+\beta} & \text { if } r_{2}=0, \\
\frac{\beta}{\alpha+\beta} & \text { if } r_{2}=1 .\end{cases} \\
& p\left(r_{2} \mid r_{1}=1\right)= \begin{cases}\frac{\beta}{\alpha+\beta} & \text { if } r_{2}=0, \\
\frac{\alpha}{\alpha+\beta} & \text { if } r_{2}=1 .\end{cases}
\end{aligned}
$$

2) Second case (FMC2 model): The density $p\left(\boldsymbol{r}_{1}^{2}\right)$ of $P_{\left(\boldsymbol{R}_{1}^{2}\right)}$ w.r.t. ${ }_{5} \nu \otimes \nu$ is of the form:

$$
\begin{aligned}
& p(0,0)=p(1,1)=\alpha, \\
& p(1,0)=p(0,1)=\beta, \\
& \gamma \text { for } r_{1}, r_{2} \in[0,1]^{2} \backslash\{0,1\}^{2} \text { and }-\delta \leq r_{2}-r_{1} \leq \delta, \\
& 0 \text { elsewhere, }
\end{aligned}
$$

with $\alpha, \beta \geq 0,0 \leq \delta<\frac{1}{2}$, and under the constraint that $\iint_{0}^{1} p\left(r_{1}, r_{2}\right) d \nu\left(r_{1}\right) d \nu\left(r_{2}\right)=1$. A possible shape for this density is illustrated in Fig. 2 By varying $\delta$, this model allows expressing transient fuzzy changes.
Remark 3. If $\alpha+\beta=\frac{1}{2}$, then $\gamma=0$, and the joint law is only made of the four Dirac's distributions at the four corners, which gives a CGOMSM.

The density $p\left(r_{1}\right)$ of $P_{\left(R_{1}\right)}$ is computed as follows:

$$
p\left(r_{1}\right)= \begin{cases}\alpha+\beta+\gamma \delta & \text { if } r_{1}=0, \\ \gamma\left(\delta+r_{1}+1\right) & \text { if } \left.\left.r_{1} \in\right] 0, \delta\right], \\ 2 \gamma \delta & \text { if } \left.r_{1} \in\right] \delta, 1-\delta[ \\ \gamma\left(2+\delta-r_{1}\right) & \text { if } r_{1} \in[1-\delta, 1[ \\ \alpha+\beta+\gamma \delta & \text { if } r_{1}=1 .\end{cases}
$$

Since $\int_{0}^{1} p\left(r_{1}\right) d \nu\left(r_{1}\right)=1$, we get

$$
\beta=\frac{1-\gamma M}{2}-\alpha
$$

with $M=\delta(6-\delta)$, and under the constraint that $\gamma \leq \frac{1-2 \alpha}{M}$. Hence this model is only parametrized by $(\alpha, \gamma, \delta)$.

The limit proportion of hard data $\left(p_{H}\right)$ to fuzzy ones $\left(p_{F}\right)$ in a sampled sequence is

$$
\begin{aligned}
& p_{H}=p(0)+p(1)=2(\alpha+\beta+\gamma \delta), \\
& p_{F}=1-p_{H}=\gamma \delta(4-\delta) .
\end{aligned}
$$

The density $p\left(r_{2} \mid r_{1}\right)$ of distribution $P_{R_{2} \mid R_{1}}$, w.r.t. $\nu$, is the ratio between the joint density and the marginal density. Similarly to the first case, we have to distinguish between different configurations, according to the value of $r_{1}$

$$
\begin{aligned}
& p\left(r_{2} \mid r_{1}=0\right)= \begin{cases}\frac{\alpha}{\alpha+\beta+\gamma \delta} & \text { if } r_{2}=0, \\
\frac{\gamma}{\alpha+\beta+\gamma \delta} & \text { if } \left.\left.r_{2} \in\right] 0, \delta\right], \\
\frac{\beta}{\alpha+\beta+\gamma \delta} & \text { if } r_{2}=1, \\
0 & \text { elsewhere. }\end{cases} \\
&\left.\left.p\left(r_{2} \mid r_{1} \in\right] 0, \delta\right]\right)= \begin{cases}\frac{1}{\delta+r_{1}+1} & \text { if } r_{2}=0, \\
\frac{1}{\delta+r_{1}+1} & \text { if } \left.\left.r_{2} \in\right] 0, r_{1}+\delta\right], \\
0 & \text { elsewhere. }\end{cases}
\end{aligned}
$$

$$
\begin{aligned}
& p\left(r_{2} \mid r_{1} \in\right] \delta, 1-\delta[)= \begin{cases}\frac{1}{2 \delta} & \text { if } \left.r_{2} \in\right] r_{1}-\delta, r_{1}+\delta[, \\
0 & \text { elsewhere. }\end{cases} \\
& p\left(r_{2} \mid r_{1} \in\left[1-\delta, 1[)= \begin{cases}\frac{1}{2+\delta-r_{1}} & \text { if } r_{2} \in\left[r_{1}-\delta, 1[,\right. \\
\frac{1}{2+\delta-r_{1}} & \text { if } r_{2}=1, \\
0 & \text { elsewhere. }\end{cases} \right.\right.
\end{aligned}
$$

$$
p\left(r_{2} \mid r_{1}=1\right)= \begin{cases}\frac{\beta}{\alpha+\beta+\gamma \delta} & \text { if } r_{2}=0 \\ \frac{\gamma}{\alpha+\beta+\gamma \delta} & \text { if } r_{2} \in[1-\delta, 1[ \\ \frac{\alpha}{\alpha+\beta+\gamma \delta} & \text { if } r_{2}=1 \\ 0 & \text { elsewhere. }\end{cases}
$$

Remark 4. In the two examples of fuzzy Markov models, we assume that the distribution $P_{\left(R_{1}, R_{2}\right)}$ is defined by four masses on the corners, -i.e. at locations $\{0,1\} \times\{0,1\}$-, and a density on the remaining $[0,1]^{2} \backslash\{0,1\}^{2}$. It is to say that we assume the distributions on the sides of the square $[0,1] \times[0,1]$, -i.e. the distributions $P_{(0, R)}$, $P_{(1, R)}, P_{(R, 0)}$ and $P_{(R, 1)}$ for $R$ in $[0,1]-$, to be identical to the inner density of the square. This is a particular case, used here to simplify the parametrization, since we can set them independently. 


\section{B. Distributions of $\left(\boldsymbol{X}_{1}^{2}, \boldsymbol{Y}_{1}^{2}\right)$ conditional on $\boldsymbol{R}_{1}^{2}$}

To finalize the description of stationary $\left(\boldsymbol{X}_{1}^{N}, \boldsymbol{R}_{1}^{N}, \boldsymbol{Y}_{1}^{N}\right)$, we need to define the 4D Gaussian distributions $p\left(\boldsymbol{x}_{1}^{2}, \boldsymbol{y}_{1}^{2} \mid \boldsymbol{r}_{1}^{2}\right)=p\left(\boldsymbol{z}_{1}^{2} \mid \boldsymbol{r}_{1}^{2}\right)$ for $r_{1}, r_{2} \in[0,1]$. The means and covariance matrices of the four 'hard Gaussians' corresponding to $r_{1}=i, r_{2}=j$, with $i, j \in\{0,1\}$, are given by

$$
\begin{aligned}
& \mu_{i, j}=\mathbb{E}\left[\begin{array}{l}
\boldsymbol{z}_{1} \\
\boldsymbol{z}_{2}
\end{array} \mid r_{1}=i, r_{2}=j\right] \\
& =\left[\begin{array}{l|l}
\mathbb{E}\left[\begin{array}{l|l}
x_{1} & r_{1}=i \\
y_{1} &
\end{array}\right] \\
\mathbb{E}\left[\begin{array}{l}
x_{2} \\
y_{2}
\end{array} r_{2}=j\right]
\end{array}\right]=\left[\begin{array}{l}
\boldsymbol{M}_{i} \\
\boldsymbol{M}_{j}
\end{array}\right],
\end{aligned}
$$

and

$$
\boldsymbol{\Gamma}_{i, j}=\left[\begin{array}{cccc}
\left(\sigma_{i}^{X}\right)^{2} & b_{i} & a_{i j} & d_{i j} \\
b_{i} & \left(\sigma_{i}^{Y}\right)^{2} & e_{i j} & c_{i j} \\
a_{i j} & e_{i j} & \left(\sigma_{j}^{X}\right)^{2} & b_{j} \\
d_{i j} & c_{i j} & b_{j} & \left(\sigma_{j}^{Y}\right)^{2}
\end{array}\right]
$$

with $d_{i j}=b_{i} c_{i j}$ in order to verify $(5$, i.e. for the model to be a CGOMSM.

The mean of a 'fuzzy Gaussians' with $\left.r_{1} \in\right] 0,1[$ is defined by linear interpolation of $\boldsymbol{M}_{i}$ and $\boldsymbol{M}_{j}$ :

$$
\boldsymbol{M}_{r_{1}}=\left(1-r_{1}\right) \boldsymbol{M}_{0}+r_{1} \boldsymbol{M}_{1},
$$

and its covariance matrix with $\left.r_{1}, r_{2} \in\right] 0,1[$ is defined by bi-linear interpolation of the 'hard covariance matrices' $\boldsymbol{\Gamma}_{i, j}$ :

$\boldsymbol{\Gamma}_{\boldsymbol{r}_{1}^{2}}=\left(1-r_{1}\right)\left(1-r_{2}\right) \boldsymbol{\Gamma}_{0,0}+r_{1} r_{2} \boldsymbol{\Gamma}_{1,1}+r_{1}\left(1-r_{2}\right) \boldsymbol{\Gamma}_{1,0}+r_{2}\left(1-r_{1}\right) \boldsymbol{\Gamma}_{0,1}$.

Then, according to 2, we have

$$
\boldsymbol{Z}_{n+1}=\boldsymbol{A}\left(\boldsymbol{r}_{n}^{n+1}\right) \boldsymbol{Z}_{n}+\boldsymbol{B}\left(\boldsymbol{r}_{n}^{n+1}\right) \boldsymbol{W}_{n+1}+\boldsymbol{N}\left(\boldsymbol{r}_{n}^{n+1}\right),
$$

15 with $\boldsymbol{N}\left(\boldsymbol{r}_{n}^{n+1}\right)=\boldsymbol{M}_{r_{n+1}}-\boldsymbol{A}\left(\boldsymbol{r}_{n}^{n+1}\right) \boldsymbol{M}_{r_{n}}$, and

$$
\begin{gathered}
\boldsymbol{A}\left(\boldsymbol{r}_{n}^{n+1}\right)=\left[\begin{array}{ll}
a_{\boldsymbol{r}_{n}^{n+1}} & e_{\boldsymbol{r}_{n}^{n+1}} \\
d_{\boldsymbol{r}_{n}^{n+1}} & c_{\boldsymbol{r}_{n}^{n+1}}
\end{array}\right]\left[\begin{array}{cc}
\left(\sigma_{r_{n}}^{X}\right)^{2} & b_{r_{n}} \\
b_{r_{n}} & \left(\sigma_{r_{n}}^{Y}\right)^{2}
\end{array}\right]^{-1}, \\
\boldsymbol{B}\left(\boldsymbol{r}_{n}^{n+1}\right)=\left[\begin{array}{cc}
\left(\sigma_{r_{n+1}}^{X}\right)^{2} & b_{r_{n+1}} \\
b_{r_{n+1}} & \left(\sigma_{r_{n+1}}^{Y}\right)^{2}
\end{array}\right]^{-1} \\
-\boldsymbol{A}\left(\boldsymbol{r}_{n}^{n+1}\right)\left[\begin{array}{ll}
a_{\boldsymbol{r}_{n}^{n+1}} & d_{\boldsymbol{r}_{n}^{n+1}} \\
e_{\boldsymbol{r}_{n}^{n+1}} & c_{\boldsymbol{r}_{n}^{n+1}}^{n}
\end{array}\right] .
\end{gathered}
$$

Also, for the later use, note that, according to [43, we have

$$
\begin{gathered}
p\left(\boldsymbol{z}_{n+1} \mid \boldsymbol{z}_{n}, \boldsymbol{r}_{n}^{n+1}\right) \\
=\mathcal{N}\left(\boldsymbol{A}\left(\boldsymbol{r}_{n}^{n+1}\right)\left(\boldsymbol{z}_{n}-\boldsymbol{M}_{r_{n}}\right)+\boldsymbol{M}_{r_{n+1}}, \boldsymbol{B}\left(\boldsymbol{r}_{n}^{n+1}\right) \boldsymbol{B}^{\boldsymbol{\top}}\left(\boldsymbol{r}_{n}^{n+1}\right)\right)(46)
\end{gathered}
$$

Remark 5. Fuzzy means (41) and variances (42) can be seen as linear interpolations of hard ones. Other kind of interpolations can

\section{EXPERIMENTAL STUDIES}

Experiments below report results of restoration on simulated data -using the two joint laws presented in the previous Section-, in order to measure the quality of the OFSRF restoration algorithm, and the influence of the number of discretization steps $F$ in approximation of integrals. Comparison is also performed with the 'hard' CGOMSM to measure the error in using this model when there exist transient changes in data.

\section{A. Simulations of a CGOFMSM sample}

To simulate a CGOFMSM $\left(\boldsymbol{X}_{1}^{N}, \boldsymbol{R}_{1}^{N}, \boldsymbol{Y}_{1}^{N}\right)$ sample, we first simulate a fuzzy Markov chain $\boldsymbol{r}_{1}^{N}$, and then simulate the observations and states $\left(\boldsymbol{y}_{1}^{N}, \boldsymbol{x}_{1}^{N}\right)$.

To draw a sample from the fuzzy Markov chain, we first simulate $r_{1}$ using $p\left(r_{1}\right)$, and then, for each $n=1, \ldots, N-1, r_{n+1}$ is obtained from $p\left(r_{n+1} \mid r_{n}\right)$, which is equal to $p\left(r_{2} \mid r_{1}\right)$. For each $r_{n+1}$ to be simulated, we first decide if the jump will be a 'hard' one or a 'fuzzy' one. To do so, a draw is performed beforehand according to the proportion of hard and fuzzy jumps. Let us explain in detail the simulation process based on the FMC2 model, the procedure is the same for the FMC1 model:

- Simulation of $r_{1}$. First, make a draw according to the proportion in eq. (33) to determine if the sample will be a 'hard' one or a 'fuzzy' one.

- If it is hard, then, using eq. (31), make a draw according to the probability vector $\left[\frac{1}{2}, \frac{1}{2}\right]$, to determine if the sample is ' 0 ' or ' 1 '.

- If it is fuzzy, then make a draw according to the density specified by the three equations in 31 corresponding to $\left.r_{1} \in\right] 0,1[$. The target density is not trivial because of the slopes in its shape, but most of specialized random number generation libraries offer a solution for sampling such density (e.g. library 'scipy' in Python language).

- Simulation of $r_{n+1}$, knowing $r_{n}$. Let us assume $r_{n}=0$, the other possible values of $r_{n}$ can be processed similarly.

- According to eq. (34), first make a draw according to the probability vector $\left[\frac{\alpha}{S}, \frac{\beta}{S}, \frac{\gamma}{S}\right]$, with $S=\alpha+\beta+\gamma \delta$, to determine if $r_{n+1}$ will be ' 0 ', ' 1 ' or 'fuzzy'.

- If it is fuzzy, then make a uniform draw on $] 0, \delta[$ to get $r_{n+1}$.

Fig. 3 shows two excerpts of simulated trajectories corresponding to the FMC1 and FMC2 models (green plain line).

Then, knowing $\left(r_{n}, r_{n+1}\right)$ and $\left(x_{n}, y_{n}\right)$, each pair $\left(y_{n+1}, x_{n+1}\right)$ is sampled from Gaussian distributions 46 whose parameters are obtained by eq. 41 and 42. For experiments conducted hereafter, we set the 'hard' mean vectors and covariance matrices to be

$$
\boldsymbol{M}_{0}=\left[\begin{array}{l}
0 \\
3
\end{array}\right], \quad \boldsymbol{M}_{1}=\left[\begin{array}{l}
0 \\
0
\end{array}\right] .
$$

and

$$
\begin{aligned}
\boldsymbol{\Gamma}_{0,0} & =\left[\begin{array}{cccc}
0.5 & 0.3 & 0.1 & 0.06 \\
0.3 & 1.0 & 0.35 & 0.4 \\
0.1 & 0.35 & 0.5 & 0.3 \\
0.06 & 0.4 & 0.3 & 1.0
\end{array}\right] \\
\boldsymbol{\Gamma}_{0,1} & =\left[\begin{array}{cccc}
0.5 & 0.3 & 0.5 & 0.14 \\
0.3 & 1.0 & 0.33 & 0.6 \\
0.5 & 0.33 & 0.75 & 0.3 \\
0.14 & 0.6 & 0.3 & 0.5
\end{array}\right] \\
\boldsymbol{\Gamma}_{1,0} & =\left[\begin{array}{cccc}
0.75 & 0.3 & 0.1 & 0.24 \\
0.3 & 0.5 & 0.35 & 0.4 \\
0.1 & 0.35 & 0.5 & 0.3 \\
0.24 & 0.4 & 0.3 & 1.0
\end{array}\right] \\
\boldsymbol{\Gamma}_{1,1} & =\left[\begin{array}{cccc}
0.75 & 0.3 & 0.5 & 0.18 \\
0.3 & 0.5 & 0.33 & 0.3 \\
0.5 & 0.33 & 0.75 & 0.3 \\
0.18 & 0.3 & 0.3 & 0.5
\end{array}\right] .
\end{aligned}
$$




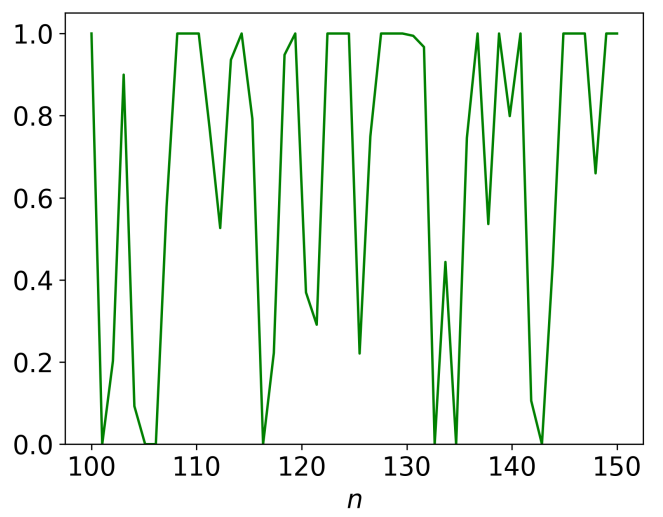

(a) FMC1 model

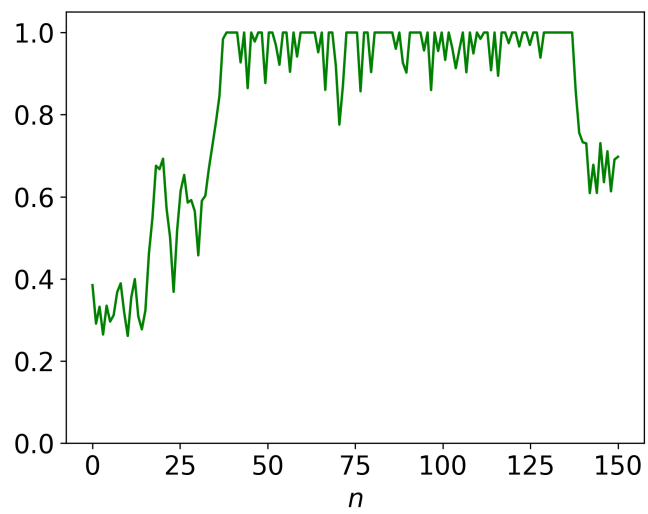

(b) FMC2 model

Fig. 3. Two excerpt of trajectories simulated from the two fuzzy models with the parameters of Figure 2

\section{B. Restoration results}

The restoration of simulated data is performed according to the OFSRF algorithm detailed in Section IV from simulated observations only. Fig. 4 shows an example of the restoration of $\boldsymbol{r}_{1}^{N}$ and $\boldsymbol{x}_{1}^{N}$ for

5 the FMC2 law and when the number of discrete fuzzy jumps are set to $F=5$. The restoration of $\boldsymbol{r}_{1}^{N}$ was obtained by applying Maximum Posteriori Mode (MPM) principle:

- If $p\left(r_{n}=0 \mid \boldsymbol{y}_{1}^{n}\right)+p\left(r_{n}=1 \mid \boldsymbol{y}_{1}^{n}\right)>0.5$ then the restoration will be "hard", else it will be "fuzzy".

- If the restoration is "hard", set $\hat{r}_{n}$ to be " 0 " if $p\left(r_{n}=0 \mid \boldsymbol{y}_{1}^{n}\right)>$ $p\left(r_{n}=1 \mid \boldsymbol{y}_{1}^{n}\right)$, else set $\hat{r}_{n}$ to be "1";

- If the restoration is "fuzzy", set $\hat{r}_{n}$ to the discrete fuzzy jump which maximizes $p\left(r_{n} \mid \boldsymbol{y}_{1}^{n}\right)$.

Note however that the restoration of $\boldsymbol{r}_{1}^{N}$ is only performed for 15 illustration purpose and is not required for the restoration of $\boldsymbol{x}_{1}^{N}$. We can observe the numerical effect of $F$ with the presence of stair-steps in the restoration of jumps in (b). Fig. (a) shows the restoration of $\boldsymbol{x}_{1}^{N}$ assuming that the jumps are known (i.e. using the simulated fuzzy Markov chain for $\boldsymbol{r}_{1}^{N}$ ). Fig. (c) assumes that

20 the jumps are unknown (OFSRF algorithm). Also, fig. (c) allows to observe the restoration difference between the 'classic' CGOMSM and the 'fuzzy' CGOFMSM, the latter follows the simulated states better than the first one for $n \leq 30$. This behaviour must be compared with that of the fuzzy jumps in Fig. (b) which shows a large difference 25 in the restoration of jumps when $n \leq 30$.

To measure the quality of restorations with respect to $F$, we compute the mean MSE of 50 independent experiments of $N=300$ samples, for the FMC1 Markov law. Fig. 5 shows the MSE evolution

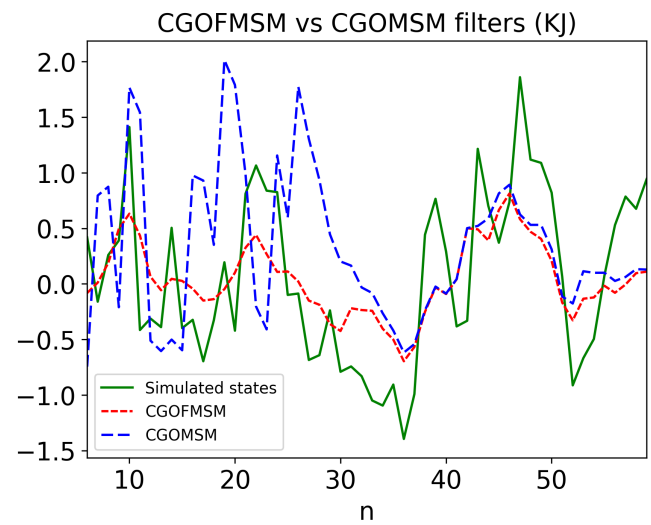

(a) Restoration of states with KJ. The MSEs for CGOFMSM and CGOMSM are 0.35 and 0.75 .

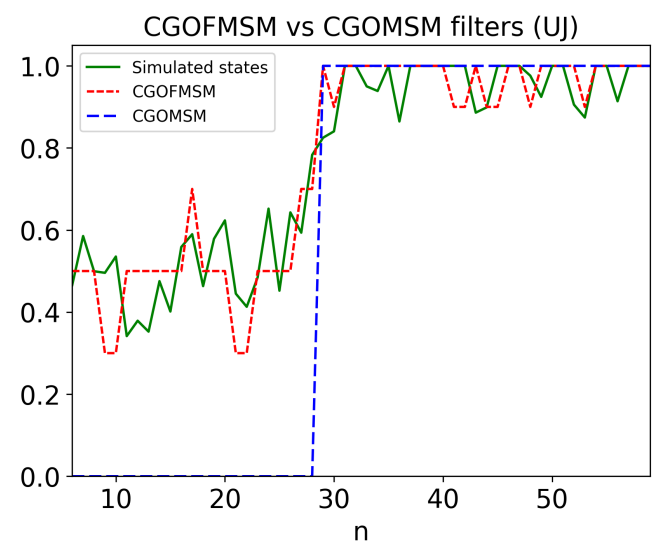

(b) MPM restoration of fuzzy jumps. The MSEs for CGOFMSM and CGOMSM are 0.03 and 0.11 .

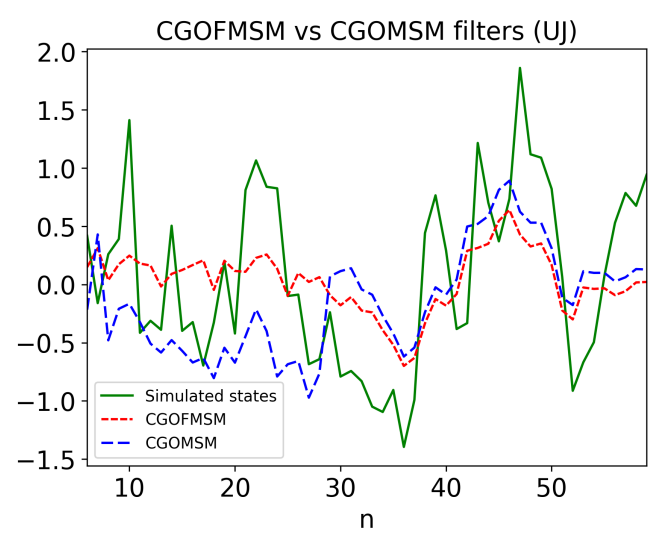

(c) Restoration of states with UJ. The MSEs for CGOFMSM and CGOMSM are 0.47 and 0.54 .

Fig. 4. Comparison of restoration of jumps and states when $F=5$, for simulated data from the FMC2 law with the same parameters as in Figure 2 $(N=80)$. 'KJ' stands for known jumps and 'UJ' for unknown jumps. The difference between the hard filter and the fuzzy one is clearly visible in (c), for $n<30$

for increasing values of $F$ for both $\boldsymbol{x}_{1}^{N}$ and $\boldsymbol{r}_{1}^{N}$. The result of applying the CGOMSM filter on the data is reported in the same graph (horizontal black dotted line, denoted as 'Hard filter - UJ'). The parameters used for the hard filters are the same than the ones used for the fuzzy filter, except that we 'harden' the Markov laws by integrating the fuzzy laws on all 4 quadrants of $[0,1]$ to get 

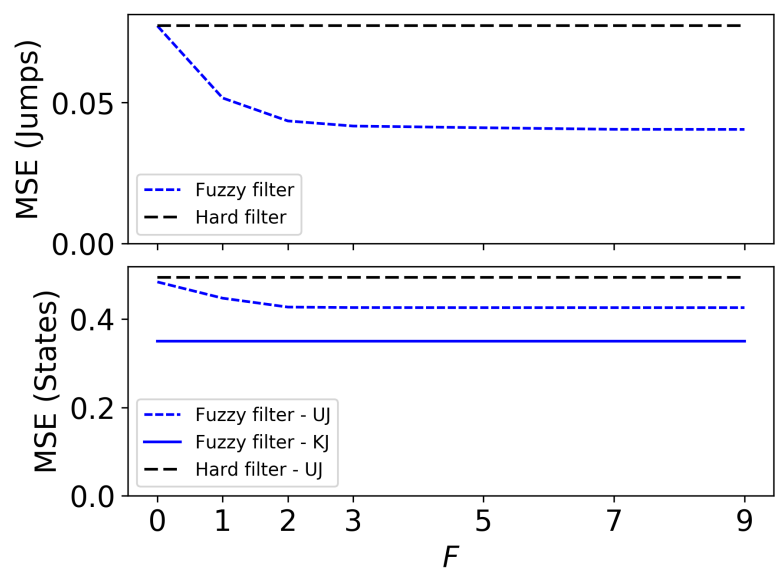

Fig. 5. Evolution of jump (up) and state (down) MSEs according to the value of the discrete fuzzy steps $F$ (means of 50 experiments of $N=300$ samples) for the FMC1 Markov law. Jumps MSEs were computed from a MPM classification. Parameters: $\alpha=0.07, \eta=0.16, \delta=0.05(\beta=0.158$, $\left.p_{H}=0.69\right)$.

\begin{tabular}{l|ccccc} 
\% of 'hard' samples - FMC1 & $\mathbf{4 8 \%}$ & $\mathbf{5 8 \%}$ & $\mathbf{6 7 \%}$ & $\mathbf{7 5 \%}$ & $\mathbf{9 3 \%}$ \\
\hline jumps MSE (CGOFMSM) & 0.051 & 0.046 & 0.039 & 0.034 & 0.017 \\
states MSE (CGOFMSM) & 0.475 & 0.446 & 0.431 & 0.415 & 0.368 \\
\hline jumps MSE (CGOMSM) & 0.126 & 0.094 & 0.074 & 0.057 & 0.020 \\
states MSE (CGOMSM) & 0.599 & 0.535 & 0.500 & 0.468 & 0.386 \\
\hline
\end{tabular}

\begin{tabular}{l|ccccc} 
\% of 'hard' samples - FMC2 & $\mathbf{4 8 \%}$ & $\mathbf{6 2 \%}$ & $\mathbf{7 5 \%}$ & $\mathbf{8 7 \%}$ & $\mathbf{1 0 0 \%}$ \\
\hline jumps MSE (CGOFMSM) & 0.030 & 0.026 & 0.021 & 0.014 & 0.012 \\
states MSE (CGOFMSM) & 0.442 & 0.442 & 0.420 & 0.399 & 0.37 \\
\hline jumps MSE (CGOMSM) & 0.070 & 0.040 & 0.033 & 0.021 & 0.011 \\
states MSE (CGOMSM) & 0.538 & 0.479 & 0.457 & 0.422 & 0.374 \\
\hline
\end{tabular}

TABLE I

EVOLUTION OF MSEs $w$.r.t. TO THE PERCENTAGE OF HARD DATA IN THE SAMPLES FOR THE FCM1 $(F=4)$ AND FMC2 $(F=10)$ MODELS. JUMPS MSES WERE COMPUTED FROM A MPM CLASSIFICATION. RESULTS ARE MEANS OF 20 INDEPENDENT EXPERIMENTS OF $N=1000$ DATA.

$P\left(R_{1}, R_{2}\right)$. In this figure, we can observe that the excess error over the model with known jumps is halved. Also, the state MSE reaches its minimum when $F>3$; this value depends on the fuzzy Markov model and on its parameters. According to some other experiments 5 not reported here, $F$ is always kept relatively small (typically $F \leq 5$ ), which is of interest since the larger $F$ is, the more the computing time increases. Indeed, the complexity is linear w.r.t. to $F$, which is to say that the computational burden of CGOFMSM is approximately the same as the one of CGOMSM with $2+F$ jumps.

The last experiment, whose results are reported in Table I for the FMC1 and FMC2 laws, shows the restoration MSE for the CGOMSM and the CGOFMSM filters when the number of fuzzy samples in the simulated data is decreasing (by adjusting parameter' values). We can observe that, for both fuzzy Markov models, the hard filter reaches

15 the performance of the fuzzy filter only when the percent of hard jumps is near $100 \%$. Elsewhere the fuzzy filter provides lower MSE, and the difference can be very large for hard sample rates lower than $50 \%$. This result confirms the interest of the fuzzy filter in comparison with the hard one in the presence of transient changes in observation data.

\section{ILLUSTRATION ON REAL DATA}

This section intends to illustrate the behaviour of the proposed algorithm when confronted to real data. The experimental data are time series representing the energy power (in kilo-Watt) consumed by

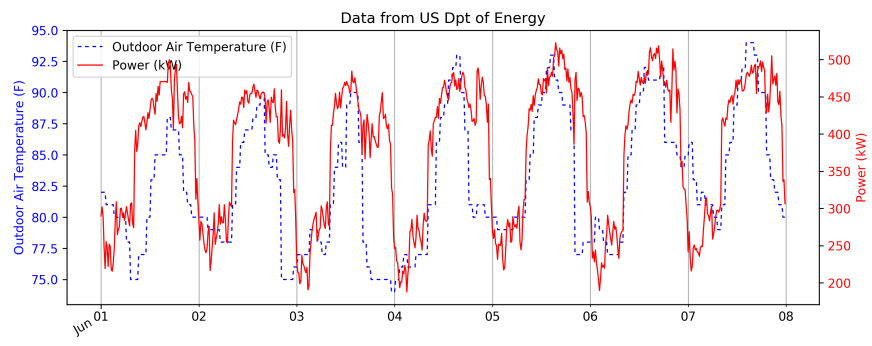

Fig. 6. Outdoor temperature (red) and energy power consumed (blue) by some building during the first week of June, 2010 (source: American DOE).

some building along with the outdoor temperature (in Fahrenheit) ${ }^{1}$ We try to understand to what extend the fuzzy model is able to infer the outdoor temperature from the consumed energy for some building during the first week of June 2010, $c f$. Fig. 6

Thus, energy consumption is considered as the observation $\left(\boldsymbol{Y}_{1}^{N}\right)$, and the outside air temperature as the state $\left(\boldsymbol{X}_{1}^{N}\right)$, to be estimated, with $N=672$. The fuzzy filter requires to know the jumps $\boldsymbol{R}_{1}^{N}$ to estimate the best suited parameters for data. Regardless the data, we have fixed that the lowest temperature appears between $1 \mathrm{am}$ and 5 am (corresponding to hard jump '0'), and that the highest appear between $1 \mathrm{pm}$ and $5 \mathrm{pm}$ (corresponding to hard jump '1'). Between these ranges, temperatures increase and decrease linearly and are represented by the fuzzy nature of the jumps considered in this model. The shape of the observations suggests that a fuzzy model is better suited than a hard model (we choose to use the FMC1 model). From this pseudo ground-truth for jumps, using classical empirical estimators, it is possible to estimate all the required parameters of the model: on the one hand, mean values and covariance matrices of $\left(\boldsymbol{X}_{1}^{N}, \boldsymbol{Y}_{1}^{N}\right)$ conditionally en jumps for the two hard jumps, and, on the other hand, the $\alpha, \beta, \eta$ and $\delta$ parameters required to define the law of $\boldsymbol{R}_{1}^{N}$.

The results of fuzzy and hard filtering are reported in cyan in Fig. 7 and 8 respectively, with the measured air temperature given in red. For the fuzzy filtering, we considered $F=5$ because it gives good results while maintaining low computation times. The MSE of estimated states with respect to the true outdoor air temperature is 8701 for the hard model, and 6759 for the fuzzy model. Regarding the jumps, the MSE is 0.13 for the hard model, and 0.07 for the fuzzy one. The better results obtained with the fuzzy model w.r.t. the hard one are illustrated by both the estimated jumps and the estimated outdoor air temperature.

\section{CONCLUSION}

We proposed a new jump Markov model made of a triplet random process (observations, hidden states, hidden fuzzy switches), and designed the related optimal recursive fuzzy filter which is able to restore switches and states from observations. We called this model "Conditionally Gaussian Observed Fuzzy Markov switching Model" (CGOFMSM). The work is based on two key ideas:

- A recursive and exact filter to deal with hard jumps, called CGOMSM, is available, see [7]|-[10]. This filter only assumes the presence of a zero-term $\sqrt{5}$ in the transition matrix of the very general Conditionally Gaussian Markov Switching model defined by 2.

${ }^{1}$ This time series, collected by the American Department of Energy, is opendata and can be downloaded for free from https://openei.org/datasets/dataset/ consumption-outdoor-air-temperature-11-commercial-buildings $\quad$ The experiments focus on building \#5. 


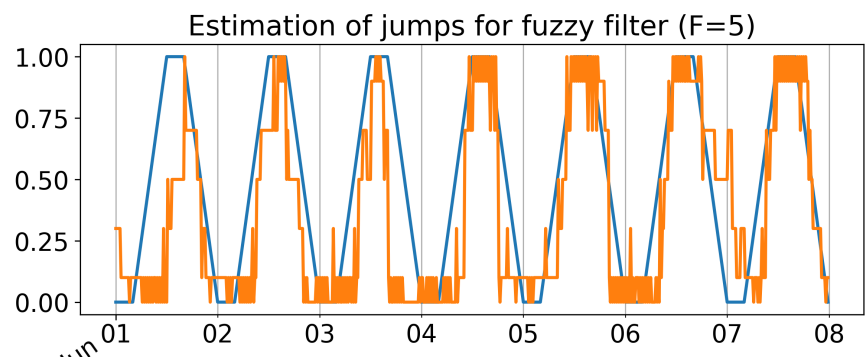

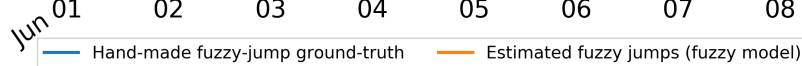

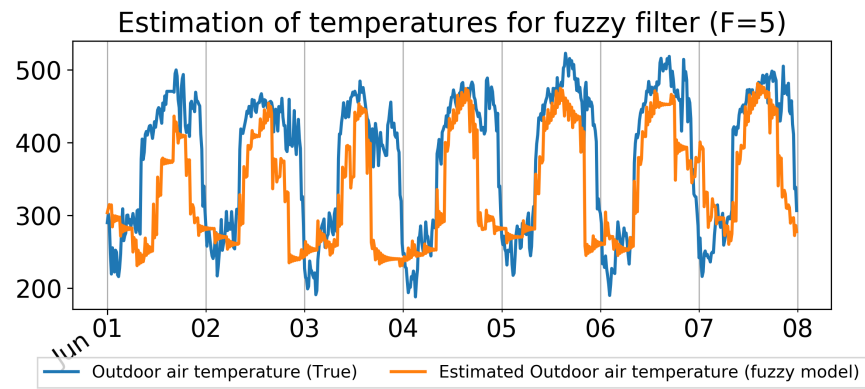

Fig. 7. Filtering result with estimated jumps (up) and estimated states (down) for the fuzzy model with $F=5$. The MSE for jumps is 0.07 , while the MSE for states is 6759 .

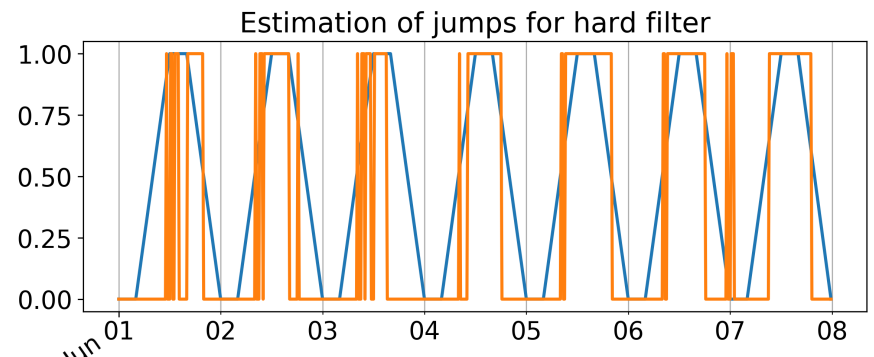

jun _ Hand-made fuzzy-jump ground-truth — Estimated fuzzy jumps (hard model)

Estimation of temperatures for hard filter

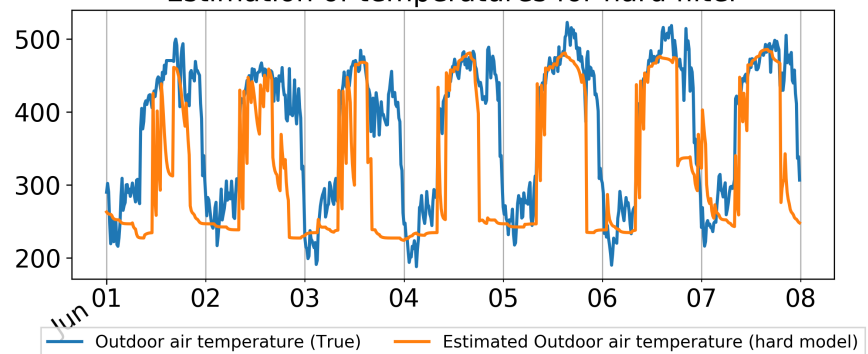

Fig. 8. Filtering result with estimated jumps (up) and estimated states (down) for the hard model. The MSE for jumps is 0.13 , while the MSE for states is 8701.

- The definition of a mixed measure including two Dirac masses for hard classes " 0 " or " 1 " and a Lebesgue measure to deal with fuzziness. It should be noted that integral calculations required some simple and low-time consuming numerical approximation.

We showed through an experimental study that the proposed model and its filter provide interesting results in terms of data restoration accuracy. This behaviour is confirmed when the model in confronted to real data dealing with outdoor air temperature. In that case, the fuzzy jumps allow a better modelling of the increasing and decreasing o air temperature cycle during one day.

In this work, we assume scalar states and scalar observations for notation convenience ; the extension to a vectorial filter is somewhat straightforward, using matrix products. However, the extension of the filter to three and more classes is not as easy, with quite complex fuzzy Markov laws to deal with, but could be inspired from the work [22]. The next step in the development of an unsupervised parameter estimation method for this CGOFMSM-similar to the one proposed for the 'hard' model [23]- is the derivation of a fuzzy smoother for off-line processing. Application of the fuzzy model to deal with the design of a control system for road traffic congestion prediction in which traffic dynamics would be modeled by a switching regime model is another perspective for further work.

\section{APPENDIX}

Here are the detail to specify the margin $p\left(r_{1}\right)$ and the parameter $\beta$ from the joint density $p\left(r_{1}, r_{2}\right)$ defined in Section V-A1

\section{A. Calculation for margin law $p\left(r_{1}\right)$}

The density $p\left(r_{1}\right)$ of distribution $P_{R_{1}}$, w.r.t. $\nu$, is obtained by

$$
\begin{gathered}
p\left(r_{1}\right)=\int_{0}^{1} p\left(r_{1}, r_{2}\right) d \nu\left(r_{2}\right)=p\left(r_{1}, 0\right)+p\left(r_{1}, 1\right)+ \\
\underbrace{\int_{0}^{1} \eta+(\delta-\eta)\left|r_{1}-r_{2}\right| d r_{2}}_{A\left(r_{1}\right)},
\end{gathered}
$$

with

$$
A\left(r_{1}\right)=\eta+(\delta-\eta) \underbrace{\int_{0}^{1}\left|r_{1}-r_{2}\right| d r_{2}}_{B\left(r_{1}\right)=B_{1}\left(r_{1}\right)+B_{2}\left(r_{1}\right)}
$$

and

$$
\begin{aligned}
B_{1}\left(r_{1}\right) & =\int_{0}^{r_{1}}\left(r_{1}-r_{2}\right) d r_{2}=r_{1}^{2}-\frac{1}{2} r_{1}^{2}=\frac{1}{2} r_{1}^{2}, \\
B_{2}\left(r_{1}\right) & =\int_{r_{1}}^{1}\left(r_{2}-r_{1}\right) d r_{2} \\
& =\frac{1}{2}\left(1-r_{1}^{2}\right)-r_{1}\left(1-r_{1}\right)=\frac{1}{2}\left(1+r_{1}^{2}\right)-r_{1} .
\end{aligned}
$$

Thus we have $B\left(r_{1}\right)=\frac{1}{2}+r_{1}^{2}-r_{1}$, and $A\left(r_{1}\right)=\eta+(\delta-\eta)\left(\frac{1}{2}+\right.$ $\left.r_{1}^{2}-r_{1}\right)$.

So, for $r_{1}=0, p\left(r_{1}\right)=\alpha+\beta+A(O)=\alpha+\beta+\frac{\delta+\eta}{2}$, for $r_{1}=1$, $p\left(r_{1}\right)=\alpha+\beta+A(1)=\alpha+\beta+\frac{\delta+\eta}{2}$, and for $\left.r_{1} \in\right] 0,1[$,

$$
\begin{aligned}
p\left(r_{1}\right)= & \eta+(\delta-\eta) r_{1}+\eta+(\delta-\eta)\left(1-r_{1}\right)+\eta+ \\
& (\delta-\eta)\left(\frac{1}{2}+r_{1}^{2}-r_{1}\right) \\
= & 3 \eta+(\delta-\eta) \frac{3}{2}+(\delta-\eta)\left(r_{1}^{2}-r_{1}\right) \\
= & \frac{3}{2}(\delta+\eta)+(\delta-\eta)\left(r_{1}^{2}-r_{1}\right) .
\end{aligned}
$$

Hence, we get eq. 23.

\section{B. Calculation of $\beta$}

We have

$$
\begin{aligned}
\int_{0}^{1} p\left(r_{1}\right) d \nu\left(r_{1}\right) & =p(0)+p(1)+\int_{0}^{1} p\left(r_{1}\right) d r_{1} \\
& =2(\alpha+\beta)+(\delta+\eta)+ \\
& \underbrace{\int_{0}^{1} \frac{3}{2}(\delta+\eta)+(\delta-\eta)\left(r_{1}^{2}-r_{1}\right) d r_{1}}_{C=C_{1}+C_{2}}
\end{aligned}
$$


with

$$
\begin{aligned}
C_{1} & =\int_{0}^{1} \frac{3}{2}(\delta+\eta) d r_{1}=\frac{3}{2}(\delta+\eta), \\
C_{2} & =(\delta-\eta) \int_{0}^{1}\left(r_{1}^{2}-r_{1}\right) d r_{1}=-\frac{1}{6}(\delta-\eta) .
\end{aligned}
$$

Knowing that $\int_{0}^{1} p\left(r_{1}\right) d \nu\left(r_{1}\right)=1$, we get $2(\alpha+\beta)+(\delta+\eta)+$ $\frac{3}{2}(\delta+\eta)-\frac{1}{6}(\delta-\eta)=1$, and find result in eq. 24).

\section{Calculation to get eq. 16}

\section{From}

$$
\begin{array}{r}
\operatorname{Var}\left[\boldsymbol{Z}_{n+1} \mid \boldsymbol{r}_{n}^{n+1}, \boldsymbol{y}_{1}^{n}\right]=\mathbb{E}\left[\boldsymbol{Z}_{n+1} \boldsymbol{Z}_{n+1}^{\top} \mid \boldsymbol{r}_{n}^{n+1}, \boldsymbol{y}_{1}^{n}\right] \\
-\mathbb{E}\left[\boldsymbol{Z}_{n+1} \mid \boldsymbol{r}_{n}^{n+1}, \boldsymbol{y}_{1}^{n}\right] \mathbb{E}\left[\boldsymbol{Z}_{n+1}^{\top} \mid \boldsymbol{r}_{n}^{n+1}, \boldsymbol{y}_{1}^{n}\right],
\end{array}
$$

we have

$$
\begin{gathered}
\mathbb{E}\left[\boldsymbol{Z}_{n+1} \boldsymbol{Z}_{n+1}^{\top} \mid \boldsymbol{r}_{n}^{n+1}, \boldsymbol{y}_{1}^{n}\right] \\
=\boldsymbol{A}_{n+1}\left(\boldsymbol{r}_{n}^{n+1}\right) \mathbb{E}\left[\boldsymbol{Z}_{n} \boldsymbol{Z}_{n}^{\top} \mid \boldsymbol{r}_{n}^{n+1}, \boldsymbol{y}_{1}^{n}\right] \boldsymbol{A}_{n+1}^{\top}\left(\boldsymbol{r}_{n}^{n+1}\right) \\
+\boldsymbol{A}_{n+1}\left(\boldsymbol{r}_{n}^{n+1}\right) \mathbb{E}\left[\boldsymbol{Z}_{n} \mid \boldsymbol{r}_{n}^{n+1}, \boldsymbol{y}_{1}^{n}\right] \boldsymbol{N}_{n+1}^{\top}\left(\boldsymbol{r}_{n}^{n+1}\right) \\
+\boldsymbol{N}_{n+1}\left(\boldsymbol{r}_{n}^{n+1}\right) \mathbb{E}\left[\boldsymbol{Z}_{n}^{\top} \mid \boldsymbol{r}_{n}^{n+1}, \boldsymbol{y}_{1}^{n}\right] \boldsymbol{A}_{n+1}^{\top}\left(\boldsymbol{r}_{n}^{n+1}\right) \\
+\boldsymbol{B}_{n+1}\left(\boldsymbol{r}_{n}^{n+1}\right) \boldsymbol{B}_{n+1}^{\top}\left(\boldsymbol{r}_{n}^{n+1}\right)+\boldsymbol{N}_{n+1}\left(\boldsymbol{r}_{n}^{n+1}\right) \boldsymbol{N}_{n+1}^{\top}\left(\boldsymbol{r}_{n}^{n+1}\right),(
\end{gathered}
$$

and, using 15 ,

$$
\begin{gathered}
\mathbb{E}\left[\boldsymbol{Z}_{n+1} \mid \boldsymbol{r}_{n}^{n+1}, \boldsymbol{y}_{1}^{n}\right] \mathbb{E}\left[\boldsymbol{Z}_{n+1}^{\top} \mid \boldsymbol{r}_{n}^{n+1}, \boldsymbol{y}_{1}^{n}\right] \\
=\boldsymbol{A}_{n+1}\left(\boldsymbol{r}_{n}^{n+1}\right) \mathbb{E}\left[\boldsymbol{Z}_{n} \mid \boldsymbol{r}_{n}^{n+1}, \boldsymbol{y}_{1}^{n}\right] \mathbb{E}\left[\boldsymbol{Z}_{n}^{\top} \mid \boldsymbol{r}_{n}^{n+1}, \boldsymbol{y}_{1}^{n}\right] \boldsymbol{A}_{n+1}^{\top}\left(\boldsymbol{r}_{n}^{n+1}\right) \\
+\boldsymbol{A}_{n+1}\left(\boldsymbol{r}_{n}^{n+1}\right) \mathbb{E}\left[\boldsymbol{Z}_{n} \mid \boldsymbol{r}_{n}^{n+1}, \boldsymbol{y}_{1}^{n}\right] \boldsymbol{N}_{n+1}^{\top}\left(\boldsymbol{r}_{n}^{n+1}\right) \\
+\boldsymbol{N}_{n+1}\left(\boldsymbol{r}_{n}^{n+1}\right) \mathbb{E}\left[\boldsymbol{Z}_{n}^{\top} \mid \boldsymbol{r}_{n}^{n+1}, \boldsymbol{y}_{1}^{n}\right] \boldsymbol{A}_{n+1}^{\top}\left(\boldsymbol{r}_{n}^{n+1}\right) \\
+\boldsymbol{N}_{n+1}\left(\boldsymbol{r}_{n}^{n+1}\right) \boldsymbol{N}_{n+1}^{\top}\left(\boldsymbol{r}_{n}^{n+1}\right) .
\end{gathered}
$$

Substracting 477) and 48) gives 16 .

\section{REFERENCES}

[1] H. A. P. Blom and Y. Bar-Shalom, "The interacting multiple model algorithm for systems with Markovian switching coefficients," IEEE Trans. on Automatic Control, vol. 33, no. 8, pp. 780-783, Aug 1988.

[2] C. Andrieu, M. Davy, and A. Doucet, "Efficient particle filtering for jump Markov systems. Application to time-varying autoregressions," IEEE Trans. Signal Processing, vol. 51, no. 7, pp. 1762-1770, 2003.

[3] M. Arulampalam, S. Maskell, N. Gordon, and T. Clapp, "A tutorial on particle filters for online non-linear/non-Gaussian Bayesian tracking," IEEE Trans. on Signal Processing, vol. 50, no. 2, pp. 174-188, Feb. 2002.

4] O. Cappé, E. Moulines, and T. Rydén, Inference in hidden Markov models. Springer-Verlag, 2005.

[5] A. Doucet and A. Johansen, A tutorial on particle filtering and smoothing: Fifteen years later. Eds. London, U.K., Oxford Univ. Press, 2011.

[6] W. Pieczynski, "Exact filtering in conditionally Markov switching hidden linear models," Comptes Rendus Mathématique, vol. 349, no. 9, pp. 587590, 2011.

[7] S. Derrode and W. Pieczynski, "Exact fast computation of optimal filter in Gaussian switching linear systems," IEEE Signal Process. Lett., vol. 20 , no. 7, pp. 701-704, 2013.

8] N. Abbassi, D. Benboudjema, and W. Pieczynski, "Kalman filtering approximations in triplet Markov Gaussian switching models," in IEEE Workshop on Statistical Signal Processing (SSP2011), Nice, France, 2830 June, 2011.

[9] N. Abbassi, D. Benboudjema, S. Derrode, and W. Pieczynski, "Optimal filter approximations in conditionally Gaussian pairwise Markov switching models," IEEE Trans. on Automatic Control, vol. 60, no. 4 pp. 1104-1109, Apr 2015.

[10] Y. Petetin and F. Desbouvries, "A class of fast exact Bayesian filters in dynamical models with jumps," IEEE Trans. Signal Processing, vol. 62, no. 14 , pp. 3643-3653, July 2014.

[11] H. Caillol, A. Hillion, and W. Pieczynski, "Fuzzy random fields and unsupervised image segmentation," IEEE Trans. Geosc. and Rem. Sens., vol. 31 , no. 4, pp. 801-810, 1993 .
[12] C. Carincotte, S. Derrode, and S. Bourennane, "Unsupervised change detection on SAR images using fuzzy hidden Markov chains," IEEE Trans. Geosc. and Rem. Sens., vol. 44, no. 2, pp. 432-441, 2006.

[13] F. Salzenstein, C. Collet, S. L. Cam, and M. Hatt "Non-stationary fuzzy Markov chain," Patt. Recog. Lett., vol. 28, no. 16, pp. 2201-2208, 2007.

[14] F. Salzenstein and C. Collet, "Fuzzy Markov random fields versus chains for multispectral image segmentation," IEEE Trans. Pattern Anal. Mach. Intell., vol. 28, no. 11, pp. 1753-1767, 2006

[15] T. Crivelli, B. Cernuschi-Frías, P. Bouthemy, and J. Yao, "Motion textures: Modeling, classification, and segmentation using mixed-state Markov random fields," SIAM J. Imaging Sciences, vol. 6, no. 4, pp. 2484-2520, 2013.

[16] T. Crivelli, P. Bouthemy, B. Cernuschi-Frías, and J. Yao, "Simultaneous motion detection and background reconstruction with a conditional mixed-state Markov random field," Int. J. of Computer Vision, vol. 94, no. 3, pp. 295-316, 2011.

[17] X. Xie, D. Yue, and C. Peng, "Multi-instant observer design of discretetime fuzzy systems: a ranking-based switching approach," IEEE Transactions on Fuzzy Systems, vol. 25, no. 5, pp. 1281-1292, Oct 2017.

[18] F. Li, P. Shi, C. Lim, and L. Wu, "Fault detection filtering for nonhomogeneous Markovian jump systems via a fuzzy approach," IEEE Transactions on Fuzzy Systems, vol. 26, no. 1, pp. 131-141, Feb 2018.

[19] P. Skoglar, U. Orguner, D. Törnqvist, and F. Gustafsson, "Pedestrian tracking with an infrared sensor using road network information," EURASIP Journal on Advances in Signal Processing, vol. 2012, no. 1, p. 26, Feb 2012.

[20] Z. Bouyahia, H. Haddad, N. Jabeur, and S. Derrode, "Real-time traffic data smoothing from GPS sparse measures using fuzzy switching linear models," in 14th Int. Conf. on Mobile Systems and Pervasive Computing (MobiSPC 2017), Leuven, Belgium, July 24-26, 2017, pp. 143-150.

[21] K. B. Petersen and M. S. Pedersen, "The Matrix Cookbook," nov 2012, version 20121115. [Online]. Available: http://www2.imm.dtu.dk/pubdb/ p.php?3274

[22] A. Gamal-Eldin, F. Salzenstein, and C. Collet, "Hidden fuzzy Markov chain model with $K$ discrete classes," in 10th Int. Conf. on Information Science, Signal Processing and their Applications (ISSPA'10), Kuala Lumpur, Malaysia, 10-13 May 2010, pp. 109-112.

48[23] F. Zheng, S. Derrode, and W. Pieczynski, "Parameter estimation in switching Markov systems and unsupervised smoothing," IEEE Trans. Automatic Control, vol. 64, no. 4, pp. 1761-1767, Apr. 2019, doi: 10.1109/TAC.2018.2863651 